\title{
Event-triggered leader-following tracking control for multivariable multi-agent systems *
}

\author{
Yi Cheng ${ }^{a}$ and V. Ugrinovskii ${ }^{\text {a }}$ \\ ${ }^{a}$ School of Engineering and Information Technology, University of New South Wales at the Australian Defence Force \\ Academy, Canberra, ACT 2600, Australia.
}

\begin{abstract}
The paper considers event-triggered leader-follower tracking control for multi-agent systems with general linear dynamics. For both undirected and directed follower graphs, we propose event triggering rules which guarantee bounded tracking errors. With these rules, we also prove that the systems do not exhibit Zeno behavior, and the bounds on the tracking errors can be tuned to a desired small value. We also show that the combinational state required for the proposed event triggering conditions can be continuously generated from discrete communications between the neighboring agents occurring at event times. The efficacy of the proposed methods is discussed using a simulation example.
\end{abstract}

Key words: Event-triggered control, leader-follower tracking, consensus control, multi-agent systems.

\section{Introduction}

Cooperative control of multi-agent systems has received increasing attention in the past decade, see [1] and references therein. However, many control techniques developed so far rely on continuous communication between agents and their neighbors. This limits practicality of these techniques.

To address this concern, several approaches have been proposed in recent years. One approach is to apply sampled control [2]. However in sampled data control schemes control action updates continue periodically with the same frequency even after the system has reached the control goal with sufficient accuracy and no longer requires intervention from the controller. Efforts to overcome this shortcoming have led to the idea of triggered control. Self-triggered control strategies [3,4,9] employ a triggering mechanism to proactively predict the next time for updating the control input ahead of time, using the current measurements. On the other hand, event-triggered controllers $[5,6,7,8,9,10]$ trigger control input updates by reacting to excessive deviations

\footnotetext{
^ This work was supported by the Australian Research Council under the Discovery Projects funding scheme (project DP120102152). Accepted for publication in Automatica on March 14, 2016.

Email addresses: y.cheng@adfa.edu.au (Yi Cheng), v.ugrinovskii@gmail.com (V. Ugrinovskii).
}

of the decision variable from an acceptable value, i.e., when a continuously monitored triggering condition is violated. This latter approach is the main focus in this paper.

The development of event-triggered controllers remains challenging, because the agents in a multi-agent system do not have access to the complete system state information required to make decisions about control input updates. To prove the concept of eventtriggering, the early work was still assuming continuous communication between the neighboring agents $[7,9]$. To circumvent this limitation, several approaches have been proposed, e.g., see $[11,12,13,14,15,16,17]$. For instance, different from $[7,9]$ where state-dependent event triggering conditions were used, [11] proposed an event-triggered control strategy using a time-dependent triggering function which did not require neighbors' information. In [12], a state-dependent event triggering condition was employed, complemented by an iterative algorithm to estimate the next triggering time, so that continuous communications between neighboring agents were no longer needed. In [13], sampled-data event detection has been used. It must be noted that these results as well as many other results in this area were developed for multi-agent systems with single or double integrator dynamics. Most recently, similar results have been developed for multi-agent systems with general dynamics $[14,15]$ and nonlinear dynamics $[16,17]$. 
All the papers mentioned above considered the eventtriggered control problem for leaderless systems. The leader-following control is one of the important problems in cooperative control of multi-agent systems $[18,19,20,1]$, and the interest in event-based solutions to this problem is growing $[21,22,23,24]$. General multidimensional leader following problems still remain technically challenging, and the development is often restricted to the study of single or double integrator dynamics $[21,22,23,24]$. Zeno behavior presents another challenge, and is not always excluded [21,22]. Excluding Zeno behavior is an important requirement on control protocols since excessively frequent communications reduce the advantages of using the event-triggered control.

In this paper, we also consider the event-triggered leaderfollowing control problem for multi-agent systems. Unlike $[21,22,23,24]$, the class of systems considered allows for general linear dynamics. Also, the leader can be marginally stable or even unstable. For both undirected and directed system interconnections, we propose sufficient conditions for the design of controllers which guarantee that the leader tracking errors are contained within certain bounds; these bounds can be optimized by tuning the parameters of the design procedure. We also show that with the proposed eventtriggered control protocols, the system does not exhibit Zeno behavior. These results are the main contribution of the paper.

Its another contribution is the event-triggered control protocols that do not require the neighboring agents to communicate continuously. Instead, the combinational state to be used in the event triggering condition is generated continuously within the controllers, by integrating the information obtained from the neighbors during their communication events. The idea is inspired by [12], however, the procedure in [12] developed for single integrator systems cannot be applied to multiagent systems with general linear dynamics considered here, since in our case dynamics of the measurement error depend explicitly on the combinational state. Also different from [12], the proposed algorithm involves oneway communications between the neighboring agents. The combinational state is computed continuously by each agent and is broadcast to its neighbors only at the time when the communication event is triggered at this node and only in one direction. The neighbors then use this information for their own computation, and do not send additional requests to measure the combinational state. This is an important advantage of our protocol compared with eventtriggered control strategies proposed in $[12,21,23,24,30]$. In these references, when an event is triggered at one agent, it must request its neighbors for additional information to update the control signals. Owing to this, our scheme is applicable to systems with a directed graph which only involves one way communications.
In comparison with the recent work on event-triggered control for general linear systems $[14,30,15,25]$, the main distinction of our method is computing the combinational state directly using the neighbors' information. This allowed us to avoid additional sampling when checking event triggering conditions, cf. [14,30]. In contrast in [15], to avoid continuous transmission of information, each agent was equipped with models of itself and its neighbors. In [25], estimators were embedded into each node to enable the agents to estimate their neighbors' states. Both approaches make the controller rather complex, compared with our controller which does not require additional models or estimators. The leader-follower context and the treatment of both directed and undirected versions of the problem are other distinctions.

The paper is organized as follows. Section 2 includes the problem formulation and preliminaries. The main results are given in Sections 3 and 4. In Section 3 we consider the case when the system of followers is connected over a directed graph. Although these results are applicable to systems connected over an undirected graph as well, the symmetry of the graph Laplacian makes it possible to derive an alternative control design scheme in Section 4. In Section 5, the generation of the combinational state is discussed. Section 6 provides an illustrative example. The conclusions are given in Section 7.

Throughout the paper, $\Re^{n}$ and $\Re^{n \times m}$ are a real Euclidean $n$-dimensional vector space and a space of real $n \times m$ matrices. $\otimes$ denotes the Kronnecker product of two matrices. $\lambda_{\max }(\cdot)$ and $\lambda_{\min }(\cdot)$ will denote the largest and the smallest eigenvalues of a real symmetric matrix. For $q \in \Re^{n}, \operatorname{diag}\{q\}$ denotes the diagonal matrix with the entries of $q$ as its diagonal elements. $I_{N}$ is the $N \times N$ identity matrix. When the dimension is clear from the context, the subscript $N$ will be suppressed.

\section{Problem formulation and preliminaries}

\subsection{Communication graph}

Consider a communication graph $\overline{\mathcal{G}}=(\overline{\mathcal{V}}, \overline{\mathcal{E}}, \overline{\mathcal{A}})$, where $\overline{\mathcal{V}}=\{0, \ldots, N\}$ is a finite nonempty node set, $\overline{\mathcal{E}} \subseteq$ $\overline{\mathcal{V}} \times \overline{\mathcal{V}}$ is an edge set of pairs of nodes, and $\overline{\mathcal{A}}$ is an adjacency matrix. Without loss of generality, node 0 will be assigned to represent the leader, while the nodes from the set $\mathcal{V}=\{1, \ldots, N\}$ will represent the followers.

The (in general, directed) subgraph $\mathcal{G}=(\mathcal{V}, \mathcal{E}, \mathcal{A})$ obtained from $\overline{\mathcal{G}}$ by removing the leader node and the corresponding edges describes communications between the followers; the edge set $\mathcal{E} \subseteq \mathcal{V} \times \mathcal{V}$ represents the communication links between them, with the ordered pair $(j, i) \in \mathcal{E}$ indicating that node $i$ obtains information from node $j$; in this case $j$ is the neighbor of $i$. The set of neighbors of node $i$ in the graph $\mathcal{G}$ is denoted as 
$N_{i}=\{j \mid(j, i) \in \mathcal{E}\}$. Following the standard convention, we assume that $\mathcal{G}$ does not have self-loops or repeated edges. The adjacency matrix $\mathcal{A}=\left[a_{i j}\right] \in \Re^{N \times N}$ of $\mathcal{G}$ is defined as $a_{i j}=1$ if $(j, i) \in \mathcal{E}$, and $a_{i j}=0$ otherwise. Let $d_{i}=\sum_{j=1}^{N} a_{i j}$ be the in-degree of node $i \in \mathcal{V}$ and $\mathcal{D}=\operatorname{diag}\left\{d_{1}, \ldots, d_{N}\right\} \in \Re^{N \times N}$. Then $\mathcal{L}=\mathcal{D}-\mathcal{A}$ is the Laplacian matrix of the graph $\mathcal{G}$, it is symmetric when $\mathcal{G}$ is undirected.

We assume throughout the paper that the leader is observed by a subset of followers. If the leader is observed by follower $i$, then the directed edge $(0, i)$ is included in $\overrightarrow{\mathcal{E}}$ and is assigned with the weighting $g_{i}=1$, otherwise we let $g_{i}=0$. We refer to node $i$ with $g_{i} \neq 0$ as a pinned node. Let $G=\operatorname{diag}\left\{g_{1}, \ldots, g_{N}\right\} \in \Re^{N \times N}$. The system is assumed to have at least one follower which can observe the leader, hence $G \neq 0$.

In addition, we assume the graph $\mathcal{G}$ contains a spanning tree rooted at a pinned node $i_{r}$, i.e., $g_{i_{r}}>0$. Then, $-(\mathcal{L}+G)$ is a Metzler matrix. According to [28], the matrix $-(\mathcal{L}+G)$ is Hurwitz stable 1 , which implies that $-(\mathcal{L}+G)$ is diagonally stable [29]. That is, there exists a positive definite diagonal matrix $\Theta=\operatorname{diag}\left\{\vartheta_{1}, \ldots, \vartheta_{N}\right\}$ such that $H=\Theta^{-1}(\mathcal{L}+G)+(\mathcal{L}+G)^{\prime} \Theta^{-1}>0$. We will also use the following notation: $\alpha=\frac{1}{2} \lambda_{\min }(H), \vartheta_{\min }=$ $\min _{i}\left(\vartheta_{i}\right), \underline{\vartheta}=\min _{i}\left(\vartheta_{i}^{-1}\right), P=\Theta^{-1}(\mathcal{L}+G)(\mathcal{L}+G)^{\prime} \Theta^{-1}$ and $F=\left(\mathcal{L}+\stackrel{i}{G}^{\prime}(\mathcal{L}+G)\right.$.

\subsection{Problem formulation}

Consider a multi-agent system consisting of a leader agent and $N$ follower agents. Dynamics of the $i$ th follower are described by the equation

$$
\dot{x}_{i}=A x_{i}+B u_{i}
$$

where $x_{i} \in \Re^{n}$ is the state, $u_{i} \in \Re^{p}$ is the control input. Also, the dynamics of the leader agent are given by

$$
\dot{x}_{0}=A x_{0}
$$

Note that the matrix $A$ is not assumed to be Hurwitz, it can be marginally stable or even unstable.

We wish to find a distributed event-triggered control law for each follower to be able to track the leader. For each agent $i$, introduce a combinational state $z_{i}(t)$,

$$
z_{i}(t)=\sum_{j \in N_{i}}\left(x_{j}(t)-x_{i}(t)\right)+g_{i}\left(x_{0}(t)-x_{i}(t)\right) .
$$

We seek to develop a control scheme where agent $i$ updates its control input at event times, which are

\footnotetext{
1 These properties of the matrix $\mathcal{L}+G$ can be guaranteed under weaker assumptions on the graph $\mathcal{G}[28]$.
}

denoted by $t_{0}^{i}, t_{1}^{i}, \ldots$, based on samples $z_{i}\left(t_{k}^{i}\right)$ of its combinational state. The value of the combinational state is held constant between updates, thus giving rise to the measurement signal $\hat{z}_{i}(t)=z_{i}\left(t_{k}^{i}\right), \quad t \in\left[t_{k}^{i}, t_{k+1}^{i}\right)$. Based on this model, consider the following control law

$$
u_{i}(t)=-K \hat{z}_{i}(t), \quad t \in\left[t_{k}^{i}, t_{k+1}^{i}\right),
$$

where $K \in \Re^{p \times n}$ is a feedback gain matrix to be defined later. The problem in this paper is to find a control law (4) and an event triggering strategy which achieve the following leader-following property

$$
\limsup _{t \rightarrow \infty} \sum_{i=1}^{N}\left\|x_{0}(t)-x_{i}(t)\right\|^{2} \leq \Delta,
$$

where $\Delta$ is a given positive constant. Furthermore, the closed loop dynamics of the followers must not exhibit Zeno behavior with the proposed event triggering rule.

Definition 1 We say that the leader-follower system (1), (2) with a control law (4) does not exhibit Zeno behavior if over any finite time period there are only a finite number of communication events between the follower systems, i.e., for every agent $i$ the sequence of event times $t_{k}^{i}$ has the property $\inf _{k}\left(t_{k+1}^{i}-t_{k}^{i}\right)>0$.

\section{Event-triggered leader-following control under a directed graph $\mathcal{G}$}

In this section, we propose an event triggering rule and a leader-following tracking control for multi-agent systems where the followers are connected over a directed graph. Our result will involve certain symmetric positive definite matrices $R, Q$, and $Y$ related through the following Riccati inequality

$$
Y A+A^{\prime} Y-2 \vartheta_{\min } Y B R^{-1} B^{\prime} Y+Q \leq 0
$$

and constants $\omega>0$ and $\mu_{i}>0$ chosen so that $\rho_{i}=\alpha_{1}-\mu_{i}-\frac{\omega}{\alpha} \alpha_{2}>0$, where $\alpha_{1}=\frac{\lambda_{\min }(Q)}{\lambda_{\max }(Y)}$ and $\alpha_{2}=\frac{\lambda_{\max }(P) \lambda_{\max }^{2}\left(Y B R^{-1} B^{\prime} Y\right)}{\underline{v} \lambda_{\min }(Y)}$. Let $\rho_{\min }=\min _{i} \rho_{i}$ and select $\nu_{i}>0, \sigma_{i} \in\left(0, \rho_{\min }\right)$ and $\gamma>0$. Introduce the combinational state measurement error for agent $i$

$$
s_{i}(t)=\hat{z}_{i}(t)-z_{i}(t) .
$$

Theorem 1 Given $R=R^{\prime}>0, Q=Q^{\prime}>0$, suppose there exists $Y=Y^{\prime}>0$ such that (6) holds. Then under the control law (4) with $K=-\frac{1}{\alpha} R^{-1} B^{\prime} Y$, the system (1), (2) achieves the leader-follower tracking property of the form (5) with $\Delta=\frac{N \gamma}{\underline{\vartheta} \lambda_{\min }(Y) \lambda_{\min }(F) \rho_{\min }}$, if the communication events are triggered at

$$
\begin{aligned}
t_{k}^{i}=\inf \left\{t>t_{k-1}^{i}:\right. \\
\left.\quad\left\|s_{i}\right\|^{2} \geq \alpha \omega\left(\mu_{i} \vartheta_{i}^{-1} z_{i}^{\prime} Y z_{i}+\nu_{i} e^{-\sigma_{i} t}+\gamma\right)\right\} .
\end{aligned}
$$


In addition, the system does not exhibit Zeno behavior.

Remark 1 The Riccati inequality (6) is similar to the Riccati inequality employed in [15]. However, [15] considers an undirected topology, and the design uses the second smallest eigenvalue of $\mathcal{L}$. In contrast, (6) uses $\vartheta_{\text {min }}$ associated with the directed graph $\mathcal{G}$. Also, the inequality (6) is equivalent to the following $\mathrm{LMI}$ in $Y^{-1}$, which can be solved using the existing LMI solvers,

$$
\left[\begin{array}{cc}
A Y^{-1}+Y^{-1} A^{\prime}-2 \vartheta_{\min } B R^{-1} B & Y^{-1} \\
Y^{-1} & -Q^{-1}
\end{array}\right] \leq 0 .
$$

Remark 2 We note that the event triggering condition (8) involves monitoring of the combinational state $z_{i}(t)$, hence the means for generating $z_{i}(t)$ continuously are needed to implement it. A computational algorithm will be introduced later to generate the combinational state using event-triggered communications.

Proof of Theorem 1: We first prove that $\left\|z_{i}(t)\right\|$ are bounded. This fact will then be used to prove that under the proposed control law the system does not exhibit Zeno behavior. Also, the property (5) will be proved after Zeno behavior is excluded.

Define the tracking error $\varepsilon_{i}(t)=x_{0}(t)-x_{i}(t)$ at node $i$. It follows from (7) and (4) that

$$
\dot{\varepsilon}_{i}(t)=A \varepsilon_{i}(t)+B K z_{i}(t)+B K s_{i}(t) .
$$

Let the Lyapunov function candidate for the system comprised of the systems $(10)$ be $V(\varepsilon)=z^{\prime}\left(\Theta^{-1} \otimes Y\right) z$, where $z=\left((\mathcal{L}+G) \otimes I_{n}\right) \varepsilon$, and $\varepsilon=\left[\begin{array}{lll}\varepsilon_{1}^{\prime} & \ldots & \varepsilon_{N}^{\prime}\end{array}\right]^{\prime}$. Then

$$
\begin{aligned}
& \frac{d V(\varepsilon)}{d t}=2 z^{\prime}\left(\left(\Theta^{-1}(\mathcal{L}+G) \otimes Y B K\right) z\right. \\
& \left.+\left(\Theta^{-1} \otimes Y A\right) z+\left(\Theta^{-1}(\mathcal{L}+G) \otimes Y B K\right) s\right) .
\end{aligned}
$$

Since $K=-\frac{1}{\alpha} R^{-1} B^{\prime} Y$, the following inequality holds

$$
\begin{aligned}
& 2 z^{\prime}\left(\left(\Theta^{-1}(\mathcal{L}+G)\right) \otimes(Y B K)\right) z \\
& =-z^{\prime}\left(H \otimes\left(\frac{1}{\alpha} Y B R^{-1} B^{\prime} Y\right)\right) z \\
& \leq-2 \alpha z^{\prime}\left(I_{N} \otimes\left(\frac{1}{\alpha} Y B R^{-1} B^{\prime} Y\right)\right) z .
\end{aligned}
$$

Using (6), it follows from (11) and (12) that

$$
\begin{aligned}
\frac{d V(\varepsilon)}{d t} \leq & -z^{\prime}\left(\Theta^{-1} \otimes Q\right) z+\frac{1}{\alpha \omega} s^{\prime} s \\
& +\frac{\omega}{\alpha} z^{\prime}\left(P \otimes\left(Y B R^{-1} B^{\prime} Y\right)^{2}\right) z .
\end{aligned}
$$

Since the triggering condition (8) enforces the property

$$
(\alpha \omega)^{-1}\left\|s_{i}\right\|^{2} \leq \mu_{i} \vartheta_{i}^{-1} z_{i}^{\prime} Y z_{i}+\nu_{i} e^{-\sigma_{i} t}+\gamma
$$

on every interval $\left[t_{k}^{i}, t_{k+1}^{i}\right)$, then it follows from (13) that

$$
\begin{aligned}
\frac{d V(\varepsilon)}{d t} & \leq-\sum_{i=1}^{N} \rho_{i} \vartheta_{i}^{-1} z_{i}^{\prime} Y z_{i}+\sum_{i=1}^{N} \nu_{i} e^{-\sigma_{i} t}+N \gamma \\
& \leq-\rho_{\min } V(\varepsilon)+\sum_{i=1}^{N} \nu_{i} e^{-\sigma_{i} t}+N \gamma .
\end{aligned}
$$

Thus, we have

$$
\begin{gathered}
V(\varepsilon) \leq e^{-\rho_{\min } t}\left(V(\varepsilon(0))-\sum_{i=1}^{N} \frac{\nu_{i}}{\rho_{\min }-\sigma_{i}}-\frac{N \gamma}{\rho_{\min }}\right) \\
+\sum_{i=1}^{N} e^{-\sigma_{i} t} \frac{\nu_{i}}{\rho_{\min }-\sigma_{i}}+\frac{N \gamma}{\rho_{\min }} \\
\leq V(\varepsilon(0))+\sum_{i=1}^{N} \frac{\nu_{i}}{\rho_{\min }-\sigma_{i}}+\frac{N \gamma}{\rho_{\min }}=\kappa
\end{gathered}
$$

where the constant $\kappa$ depends on the initial conditions. It then follows from (17) that for all $t \geq 0$

$$
\kappa \geq V(\varepsilon)=z^{\prime}\left(\Theta^{-1} \otimes Y\right) z \geq \underline{\vartheta} \lambda_{\min }(Y) \sum_{i=1}^{N}\left\|z_{i}\right\|^{2} .
$$

This implies that for all $i,\left\|z_{i}(t)\right\|$ is bounded,

$$
\left\|z_{i}(t)\right\| \leq \sqrt{\kappa\left(\underline{\vartheta} \lambda_{\min }(Y)\right)^{-1}}=\bar{\kappa}
$$

Next, we prove that the system does not exhibit Zeno behavior. Suppose $t_{1}, t_{2}$ are two adjacent zero points of $s_{i}(t)$ on the interval $\left[t_{k}^{i}, t_{k+1}^{i}\right), t_{k}^{i} \leq t_{1}<t_{2}<t_{k+1}^{i}$. Then $\left\|s_{i}(t)\right\|>0$ for all $t \in\left(t_{1}, t_{2}\right) \subseteq\left(t_{k}^{i}, t_{k+1}^{i}\right)$, and the following inequality holds on the interval $\left(t_{1}, t_{2}\right)$

$$
\frac{d}{d t}\left\|s_{i}\right\|=\frac{d}{d t}\left(s_{i}^{\prime} s_{i}\right)^{1 / 2}=\frac{s_{i}^{\prime} \dot{s}_{i}}{\left\|s_{i}\right\|} \leq \frac{\left\|s_{i}\right\|\left\|\dot{s}_{i}\right\|}{\left\|s_{i}\right\|}=\left\|\dot{s}_{i}\right\| .
$$

Furthermore, note that on the interval $\left[t_{1}, t_{2}\right)$

$$
\dot{s}_{i}(t)=-\dot{z}_{i}(t), \quad s_{i}\left(t_{1}^{+}\right)^{2}=0 .
$$

It follows from (2) that $\forall t \in\left[t_{1}, t_{2}\right)$

$$
\begin{aligned}
& \left\|\dot{s}_{i}(t)\right\|=\left\|\sum_{j \in N_{i}}\left(\dot{x}_{j}(t)-\dot{x}_{i}(t)\right)+g_{i}\left(\dot{x}_{0}(t)-\dot{x}_{i}(t)\right)\right\| \\
& =\left\|A z_{i}(t)+B K\left(\sum_{j \in N_{i}}\left(z_{i}\left(t_{k}^{i}\right)-\hat{z}_{j}(t)\right)+g_{i} z_{i}\left(t_{k}^{i}\right)\right)\right\| \\
& \leq\|A\|\left\|s_{i}(t)\right\|+M_{k}^{i},
\end{aligned}
$$

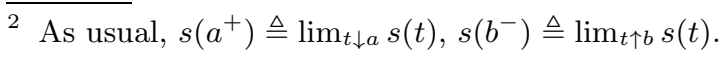


where $M_{k}^{i}=\max _{t \in\left[t_{k}^{i}, t_{k+1}^{i}\right)} \| A z_{i}\left(t_{k}^{i}\right)+B K\left(\sum_{j \in N_{i}}\left(z_{i}\left(t_{k}^{i}\right)-\right.\right.$ $\left.\left.\hat{z}_{j}(t)\right)+g_{i} z_{i}\left(t_{k}^{i}\right)\right) \|$. Hence, using (20) and (22) we obtain $\left\|s_{i}\right\| \leq \frac{M_{k}^{i}}{\|A\|}\left(e^{\|A\|\left(t-t_{1}\right)}-1\right) \leq \frac{M_{k}^{i}}{\|A\|}\left(e^{\|A\|\left(t_{k+1}^{i}-t_{k}^{i}\right)}-1\right)$

for all $t \in\left(t_{1}, t_{2}\right)$. Since $s_{i}\left(t_{1}^{+}\right)=0,(23)$ holds for all $t \in\left[t_{1}, t_{2}\right) \subseteq\left[t_{k}^{i}, t_{k+1}^{i}\right)$. The expression on the right hand side of (23) is independent of $t$; hence the above reasoning applies to all such intervals $\left[t_{1}, t_{2}\right)$. Hence, $(23)$ holds for all $t \in\left[t_{k}^{i}, t_{k+1}^{i}\right)$. Thus, from the definition of the event time $t_{k+1}^{i}$ in (8) and (23) we obtain

$\sqrt{\alpha \omega \gamma} \leq\left\|s_{i}\left(\left(t_{k+1}^{i}\right)^{-}\right)\right\| \leq \frac{M_{k}^{i}}{\|A\|}\left(e^{\|A\|\left(t_{k+1}^{i}-t_{k}^{i}\right)}-1\right)$.

According to (19), for any $k, M_{k}^{i} \leq\left(\|A\|+\left(2 d_{i}+\right.\right.$ $\left.\left.g_{i}\right)\|B K\|\right) \bar{\kappa}=\eta_{i} \bar{\kappa} \leq \bar{\eta} \bar{\kappa}$, where $\bar{\eta}=\max _{i} \eta_{i}$. Hence, it follows from (24) that

$$
t_{k+1}^{i}-t_{k}^{i} \geq \frac{1}{\|A\|} \ln \left(1+\frac{\|A\| \sqrt{\alpha \omega \gamma}}{\bar{\eta} \bar{\kappa}}\right) .
$$

Thus, the inter-event intervals are bounded from below uniformly in $k$, that is, Zeno behavior does not occur.

Since Zeno behavior has been ruled out, it follows from (16) and the rightmost inequality in (18) that for all $i$,

$$
\limsup _{t \rightarrow \infty} \sum_{i=1}^{N}\left\|z_{i}(t)\right\|^{2} \leq N \gamma\left(\underline{\vartheta} \lambda_{\min }(Y) \rho_{\min }\right)^{-1} .
$$

Since $z=\left((\mathcal{L}+G) \otimes I_{n}\right) \varepsilon$, this further implies

$$
\limsup _{t \rightarrow \infty} \sum_{i=1}^{N}\left\|\varepsilon_{i}(t)\right\|^{2} \leq \frac{N \gamma}{\underline{\vartheta} \lambda_{\min }(Y) \lambda_{\min }(F) \rho_{\min }} .
$$

I.e., (5) holds. This concludes the proof.

According to (25) and (27), the parameter $\gamma$ not only helps to exclude Zeno behavior, but also determines the upper bound of the tracking errors. We now show that after a sufficiently large time, the lower bound on the inter-event intervals becomes independent of $\gamma$. More precisely, the following statement holds.

Corollary 1 For any $\delta>0$, there exists a sufficiently large $t_{\delta}$ such that with the control law and event triggering condition proposed in Theorem 1,

$$
\begin{aligned}
& \inf _{k: t_{k}^{i}>t_{\delta}}\left(t_{k+1}^{i}-t_{k}^{i}\right) \\
& \quad \geq \frac{1}{\|A\|} \ln \left(1+\frac{\|A\| \sqrt{\alpha \omega \underline{\vartheta} \lambda_{\min }(Y) \rho_{\min }}}{\bar{\eta}(1+\delta) \sqrt{N}}\right)=\pi .
\end{aligned}
$$

Proof: According to (26), for any $\delta>0$, there exists $t_{\delta}$ such that $\left\|z_{i}(t)\right\|<(1+\delta) \sqrt{\frac{N \gamma}{\underline{\vartheta} \lambda_{\min }(Y) \rho_{\min }}} \triangleq \varpi_{1}$ for all $i$ and $t>t_{\delta}$. Therefore, for a sufficiently large $k$, $M_{k}^{i} \leq\left(\|A\|+\left(2 d_{i}+g_{i}\right)\|B K\|\right) \varpi_{1} \leq \bar{\eta} \varpi_{1}$. Then (28) follows from (24).

Remark 3 From (27), the upper bound on the tracking error depends on the parameter $\gamma$ and the size of the network $N$. Therefore, the tracking performance can be guaranteed even for larger systems, if $\gamma$ is sufficiently small. On the other hand, the lower bound on the interevent times in (25) reduces if $\gamma$ is reduced. This means that a higher tracking precision can be achieved by reducing $\gamma$, but the communications may become more frequent. However, Corollary 1 shows that when $\gamma$ is reduced, the frequency of communication events may increase only on an initial interval $\left[0, t_{\delta}\right]$, and after time $t_{\delta}$ the minimum inter-event time $\pi$ is independent of $\gamma$.

Remark 4 Selecting the parameters for the eventtriggering condition (8) involves the following steps: (a) choose matrices $Q>0$ and $R>0$ and solve the Riccati inequality (6), or equivalently the LMI (9), to obtain the matrix $Y$, then compute $\alpha_{1}$ and $\alpha_{2}$; (b) choose $\mu_{i}>0$ and $\omega>0$ to compute $\rho_{i}>0$; (c) choose $\sigma_{i} \in\left(0, \rho_{\min }\right)$; (d) based on the desired upper bound $\Delta$, select $\gamma$, see (27); (e) Lastly, choose $\nu_{i}$. Note that the term $\nu_{i} e^{-\sigma_{i} t}$ in (8) governs the triggering threshold during the initial stage of the tracking process. Thus it determines the frequency of communication events during this stage. The value of $\nu_{i}$ depends on the selected $\sigma_{i}$. If $\sigma_{i}$ is large, then typically a relatively large $\nu_{i}$ must be chosen to ensure the communication events occur less frequently.

\section{Event-triggered leader-following control under an undirected graph $\mathcal{G}$}

Although the problem for an undirected $\mathcal{G}$ can be regarded as a special case of the problem in Section 3, an independent derivation is of interest, which uses the symmetry of the matrix $\mathcal{L}+G$. Accordingly, a different event triggering condition is proposed for this case.

Theorem 2 Let $R=R^{\prime}>0, Q=Q^{\prime}>0$ be given matrices. Suppose there exists a matrix $Y=Y^{\prime}>0, Y \in$ $\Re^{n \times n}$, solving the following Riccati inequality

$$
Y A+A^{\prime} Y-2 \underline{\lambda} Y B R^{-1} B^{\prime} Y+Q \leq 0,
$$

where $\underline{\lambda}=\lambda_{\min }\left(\lambda_{i}\right)$ and $\lambda_{i}$ are the eigenvalues of $\mathcal{L}+G$. Then under the control law (4) with $K=$ $-R^{-1} B^{\prime} Y$ the system (1), (2) achieves the leaderfollower tracking property of the form (5) with $\Delta=$ $N \gamma\left(\rho \lambda_{\min }(Y) \lambda_{\min }(F)\right)^{-1}$, if the communication events 
are triggered at

$t_{k}^{i}=\inf \left\{t>t_{k-1}^{i}:\left\|z_{i}\right\|\left\|s_{i}\right\| \geq \frac{\mu_{i} z_{i}^{\prime} Q z_{i}+\nu_{i} e^{-\sigma_{i} t}+\gamma}{2 \varpi_{2}}\right\}$

here $\varpi_{2}=\lambda_{\max }(\mathcal{L}+G) \lambda_{\max }\left(Y B R^{-1} B^{\prime} Y\right), \mu_{i}, \nu_{i}, \sigma_{i}$ and $\gamma$ are positive constants chosen so that $0<\mu_{i}<1$, $\nu_{i}>0, \gamma>0$ and $\sigma_{i} \in(0, \rho)$, where $\rho=(1-$ $\left.\mu_{\max }\right) \lambda_{\min }(Q) / \lambda_{\max }(Y), \mu_{\max }=\max _{i} \mu_{i}$. In addition under this control law, Zeno behavior is ruled out:

$$
\inf _{k}\left(t_{k+1}^{i}-t_{k}^{i}\right) \geq \frac{1}{\|A\|} \ln \left(1+\frac{\|A\| \gamma}{2 \varpi_{2} \bar{\eta} \hbar^{2}}\right)
$$

here $\hbar=\sqrt{h / \lambda_{\min }(Y)}, h$ is defined in (34) below.

Remark 5 The Riccati inequality (29) in this theorem is similar to the Riccati inequality employed in [15]. However, our condition (29) depends on the smallest eigenvalue $\underline{\lambda}$ of the matrix $\mathcal{L}+G$. In contrast, in [15] the second smallest eigenvalue of the graph Laplacian matrix is required to build the consensus algorithm. When the graph topology is completely known at each node, $\underline{\lambda}$ can be readily computed. But even when the graph $\mathcal{G}$ is not known at each node, $\underline{\lambda}$ can be estimated in a decentralized manner [26]. Errors between the true eigenvalue $\underline{\lambda}$ and its estimate $\underline{\hat{\lambda}}$ can be accommodated by replacing (29) with a slightly more conservative condition. Suppose $|\underline{\lambda}-\underline{\hat{\lambda}}|<\varrho_{1}$, then the following Riccati inequality can be used in lieu of (29):

$$
Y A+A^{\prime} Y-2\left(\underline{\hat{\lambda}}-\varrho_{1}\right) Y B R^{-1} B^{\prime} Y+Q \leq 0 .
$$

Proof of Theorem 2: The proof is similar to the proof of Theorem 1 except for the procedure of obtaining an upper bound of $z_{i}(t)$. Therefore, we only outline the proof of boundedness of $z_{i}(t)$. The closed loop system consisting of error dynamics (10) is represented as

$$
\dot{\varepsilon}=\left(I_{N} \otimes A+(\mathcal{L}+G) \otimes B K\right) \varepsilon+\left(I_{N} \otimes B K\right) s,
$$

where as before $\varepsilon=\left[\begin{array}{lll}\varepsilon_{1}^{\prime} & \ldots & \varepsilon_{N}^{\prime}\end{array}\right]^{\prime}$ and $s=\left[\begin{array}{lll}s_{1}^{\prime} & \ldots & s_{N}^{\prime}\end{array}\right]^{\prime}$.

It follows from [20] that all the eigenvalues of matrix $\mathcal{L}+G$ ar positive. Let $T \in \Re^{N \times N}$ be an orthogonal matrix such that $T^{-1}(\mathcal{L}+G) T=\Lambda=\operatorname{diag}\left\{\lambda_{1}, \ldots, \lambda_{N}\right\}$. Also, let $\zeta=\left(T^{-1} \otimes I_{n}\right) \varepsilon, \zeta=\left[\begin{array}{lll}\zeta_{1}^{\prime} & \ldots & \zeta_{N}^{\prime}\end{array}\right]^{\prime}$. Using this coordinate transformation, the system (32) can be represented in terms of $\zeta$ and $s$, as

$$
\dot{\zeta}=\left(I_{N} \otimes A+\Lambda \otimes(B K)\right) \zeta+\left(T^{-1} \otimes(B K)\right) s .
$$

Consider the following Lyapunov function candidate for the system (33), $V(\zeta)=\zeta^{\prime}\left(\Lambda^{2} \otimes Y\right) \zeta$. Using (29), the coordinate transformation $\zeta=\left(T^{-1} \otimes I_{n}\right) \varepsilon$, the identity $z=\left((\mathcal{L}+G) \otimes I_{n}\right) \varepsilon, z=\left[z_{1}^{\prime}, \ldots, z_{N}^{\prime}\right]^{\prime}$, and condition (30) we can show that on every interval $\left[t_{k}^{i}, t_{k+1}^{i}\right)$,

$$
\begin{aligned}
V(\zeta) \leq & e^{-\rho t}\left(V(\zeta(0))-\sum_{i=1}^{N} \frac{\nu_{i}}{\rho-\sigma_{i}}-\frac{N \gamma}{\rho}\right) \\
& +\sum_{i=1}^{N} e^{-\sigma_{i} t} \frac{\nu_{i}}{\rho-\sigma_{i}}+\frac{N \gamma}{\rho} \\
\leq & V(\zeta(0))+\sum_{i=1}^{N} \frac{\nu_{i}}{\rho-\sigma_{i}}+\frac{N \gamma}{\rho}=h .
\end{aligned}
$$

$V(\zeta)$ can be expressed in terms of $\varepsilon$ using the inverse transformation $\zeta=\left(T^{-1} \otimes I_{n}\right) \varepsilon$ and $z=\left((\mathcal{L}+G) \otimes I_{n}\right) \varepsilon$

$$
V(\zeta)=\varepsilon^{\prime}\left((\mathcal{L}+G)^{2} \otimes Y\right) \varepsilon=z^{\prime}\left(I_{N} \otimes Y\right) z
$$

It then follows from $(35)$ that $\lambda_{\min }(Y) \sum_{i=1}^{N}\left\|z_{i}(t)\right\|^{2} \leq$ $V(\zeta)$. The rest of the proof of this theorem is similar to the proof of Theorem 1 and is omitted for brevity.

Remark 6 It can be shown that the observation made in Remark 3 applies in this case as well. The parameters in the event triggering conditions (30) can be selected following a process similar to that outlined in Remark 4.

\section{Generation of the combinational state}

To implement the event triggering conditions (8) and (30) in Theorems 1 and 2, the combinational state $z_{i}(t)$ must be known at all times. We now describe how node $i$ can generate $z_{i}(t)$ continuously using only discrete communications from its neighbors at event times. This eliminates the need for agent $i$ to monitor and communicate with its neighbors continuously.

According to (1), for $t \in\left[t_{k}^{i}, t_{k+1}^{i}\right)$, the dynamics of $x_{i}(t)$ and $x_{j}(t), j \in N_{i}$, on this interval can be expressed as

$$
\begin{aligned}
& x_{i}(t)=e^{A\left(t-t_{k}^{i}\right)} x_{i}\left(t_{k}^{i}\right)-\int_{t_{k}^{i}}^{t} e^{A(t-\tau)} B K z_{i}\left(t_{k}^{i}\right) d \tau, \\
& x_{j}(t)=e^{A\left(t-t_{k}^{i}\right)} x_{j}\left(t_{k}^{i}\right)-\int_{t_{k}^{i}}^{\hat{t}_{j}} e^{A(t-\tau)} B K z_{j}\left(t_{l}^{j}\right) d \tau \\
& -\sum_{m: t_{k}^{i}<t_{m}^{j}<t} \int_{t_{m}^{j}}^{\min \left(t, t_{m+1}^{j}\right)} e^{A(t-\tau)} B K z_{j}\left(t_{m}^{j}\right) d \tau, \\
& \hat{t}_{j}= \begin{cases}t_{l+1}^{j}, & \text { if } j \text { has at least one event on }\left[t_{k}^{i}, t\right), \\
t, & \text { otherwise, }\end{cases}
\end{aligned}
$$

where $t_{l+1}^{j}=\min \left(t_{m}^{j}: t_{m}^{j} \in\left[t_{k}^{i}, t\right)\right)$. Equation (37) accounts for the fact that agent $j$ may experience several 
events at times $t_{m}^{j}, m=l+1, \ldots$, within the time interval $\left[t_{k}^{i}, t_{k+1}^{i}\right)$. When $\left[t_{k}^{i}, t\right)$ contains no event triggered by agent $j$, the last term in (37) vanishes. Similarly, the dynamics of the tracking error $\varepsilon_{i}(t)$ can be expressed as

$$
\varepsilon_{i}(t)=e^{A\left(t-t_{k}^{i}\right)} \varepsilon_{i}\left(t_{k}^{i}\right)+\int_{t_{k}^{i}}^{t} e^{A(t-\tau)} B K z_{i}\left(t_{k}^{i}\right) d \tau .
$$

Using the notation $\Phi\left(t, t^{\prime}\right)=\int_{t}^{t^{\prime}} e^{A\left(t^{\prime}-\tau\right)} B K d \tau$, it follows from (3), (36), (37) and (38) that

$$
\begin{aligned}
& z_{i}(t)=e^{A\left(t-t_{k}^{i}\right)} z_{i}\left(t_{k}^{i}\right)+g_{i} \Phi\left(t_{k}^{i}, t\right) z_{i}\left(t_{k}^{i}\right) \\
& +\sum_{j \in N_{i}} \sum_{m: t_{k}^{i}<t_{m}^{j}<t} \Phi\left(t_{m}^{j}, \min \left(t, t_{m+1}^{j}\right)\right)\left(z_{i}\left(t_{k}^{i}\right)-z_{j}\left(t_{m}^{j}\right)\right) \\
& +\sum_{j \in N_{i}} \Phi\left(t_{k}^{i}, \hat{t}_{j}\right)\left(z_{i}\left(t_{k}^{i}\right)-z_{j}\left(t_{l}^{j}\right)\right) .
\end{aligned}
$$

According to (39), to generate $z_{i}(t)$, agent $i$ must know $z_{i}\left(t_{k}^{i}\right)$ and $z_{j}\left(t_{m}^{j}\right), t_{k}^{i}<t_{m}^{j}<t$. It has $z_{i}\left(t_{k}^{i}\right)$ in hand and thus it must only receive $z_{j}\left(t_{m}^{j}\right)$ when an event is triggered at node $j$ during $\left[t_{k}^{i}, t_{k+1}^{i}\right)$. To ensure this, we propose an algorithm to allow every agent to compute its combinational state and broadcast it to its neighbors at time instants determined by its triggering condition. This algorithm has a noteworthy feature that follows from (39) in that only one-way communications occur between the neighboring agents at the triggering time, even when the graph $\mathcal{G}$ is undirected.

Before presenting the algorithm formally, let us illustrate using (39) with an example involving three agents, $\mathbf{A}_{1}$, $\mathbf{A}_{2}$ and $\mathbf{A}_{3}$; see Fig. 1 and 2. E.g., consider the timeline in Fig. 1(b). According to the timeline of $\mathbf{A}_{2}$, an event has been triggered for $\mathbf{A}_{2}$ at time $t_{k}^{2}$. Until it receives communications from the neighbors, $\mathbf{A}_{2}$ computes $z_{2}(t)$ using (39) with the information $z_{1}\left(t_{p-1}^{1}\right)$ and $z_{3}\left(t_{q-1}^{3}\right)$ received from $\mathbf{A}_{1}$ and $\mathbf{A}_{3}$ prior time $t_{k}^{2}$. This information is used in the third line of (39). Note that $\hat{t}_{1}=\hat{t}_{3}=t$ until $\mathbf{A}_{2}$ receives the first message; the terms in the second line are zero until then. At time $t_{p}^{1}$, an event occurs at node 1 , and $\mathbf{A}_{2}$ receives the value of $\mathbf{A}_{1}$ 's combinational state, $z_{1}\left(t_{p}^{1}\right)$. From this time on, it starts using $\Phi\left(t_{p}^{1}, t\right)\left(z_{2}\left(t_{k}^{2}\right)-z_{1}\left(t_{p}^{1}\right)\right)$ in (39), until the next message arrives, this time from $\mathbf{A}_{3}$. Overall during the interval $\left[t_{k}^{2}, t_{k+1}^{2}\right), \mathbf{A}_{2}$ receives $z_{1}\left(t_{p}^{1}\right)$ and $z_{1}\left(t_{p+1}^{1}\right)$ from $\mathbf{A}_{1}$ and $z_{3}\left(t_{q}^{3}\right)$ from $\mathbf{A}_{3}$, which it uses in (39) to compute $z_{2}(t)$. When $\mathcal{G}$ is directed, $\mathbf{A}_{2}$ computes $z_{2}(t)$ in the same manner, but it only receives $z_{1}\left(t_{p}^{1}\right)$ and $z_{1}\left(t_{p+1}^{1}\right)$ from $\mathbf{A}_{1}$, as shown in Fig. 2(b).

We conclude this section by summarizing the algorithm for generating the combinational state $z_{i}(t)$ at each node.

Initialization. (a) Synchronize local clocks to set $t=$ 0 at each node, also set the event counter $k=0$,

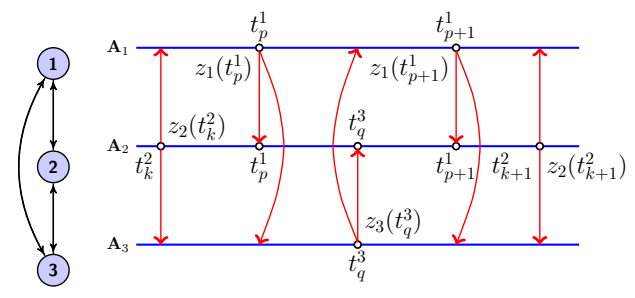

(a)

(b)

Fig. 1. Communication between followers in an undirected network: (a) The graph; (b) Communication events.

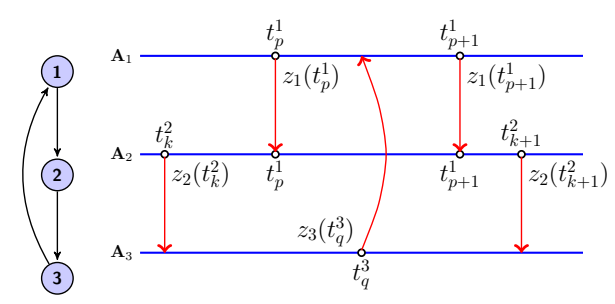

(a)

(b)

Fig. 2. Communication between followers in a directed network: (a) The graph; (b) Communication events.

the local event time record $t_{k}^{i}=0$, and the local measurement error $s_{i}(t)=0$.

(b) Receive $x_{j}(0)$ from all neighbors $j \in N_{i}$;

(c) Send $x_{i}(0)$ to agents $r$ such that $i \in N_{r}$;

(d) Compute $z_{i}(0)$ using the received $x_{j}(0), j \in N_{i}$;

(e) Receive $z_{j}(0), j \in N_{i}$, and send $z_{i}(0)$ to agents $r$ such that $i \in N_{r}$.

Do While (8) (if $\mathcal{G}$ is directed) or (30) (if $\mathcal{G}$ is undirected) is not satisfied:

(a) Compute $z_{i}(t)$ with the latest received $\hat{z}_{j}(t), j \in$ $N_{i}$, using (39), then update $s_{i}(t)$ using $(7)$;

Else

(a) Advance the event counter $k=k+1$, and set $t_{k}^{i}=t, s_{i}(t)=0$

(b) Set $z_{i}\left(t_{k}^{i}\right)=z_{i}(t)$ and send $z_{i}\left(t_{k}^{i}\right)$ to agents $r$ such that $i \in N_{r}$;

End

(c) Update the control signal $u_{i}=-K z_{i}\left(t_{k}^{i}\right)$.

As one can see, the algorithm uses only one-directional communications between agents at event times: the information is received from $j \in N_{i}$ when an event occurs at node $j$ and is sent to $r, i \in N_{r}$ when an event occurs at node $i$, e.g., see Figs. 1(b) and 2(b).

\section{Example}

Consider a system consisting of twenty identical pendulums. Each pendulum is subject to an input as shown in Fig. 3. The dynamic of the $i$-th pendulum is 


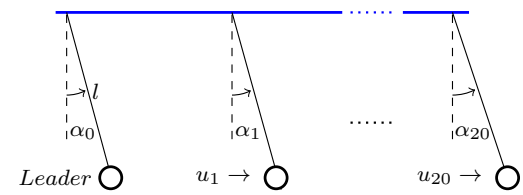

Fig. 3. The system consisting of twenty pendulums and the leader pendulum.

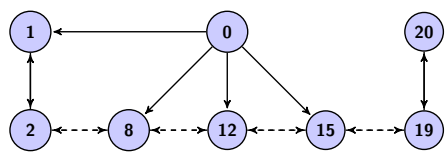

(a) Undirected follower graph

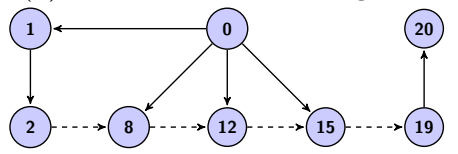

(b) Directed follower graph

Fig. 4. Communication graphs for the example.

governed by the following linearized equation

$$
m l^{2} \ddot{\alpha}_{i}=-m g l \alpha_{i}-u_{i}, \quad i=1, \ldots, 20,
$$

where $l$ is the length of the pendulum, $g=9.8 \mathrm{~m} / \mathrm{s}^{2}$ is the gravitational acceleration constant, $m$ is the mass of each pendulum and $u_{i}$ is the control torque (realized using a DC motor). In addition, consider the leader pendulum which is identical to those given and whose dynamic is described by the linearized equation

$$
m l^{2} \ddot{\alpha}_{0}=-m g l \alpha_{0}
$$

Choosing the state vectors as $x_{i}=\left(\alpha_{i}, \dot{\alpha}_{i}\right), i=0, \ldots, 20$, equations (40) and (41) can be written in the form of (1), (2), where $A=\left[\begin{array}{cc}0 & 1 \\ -g / l & 0\end{array}\right], B=\left[\begin{array}{c}0 \\ -1 /\left(m l^{2}\right)\end{array}\right]$. In this example, we let $m=1 \mathrm{~kg}, l=1 \mathrm{~m}$.

Both undirected and directed follower graphs $\mathcal{G}$ are considered in the example, shown in Fig. 4(a) and 4(b), respectively. According to Fig. 4, in both cases agents $1,8,12$ and 15 measure the leader's state, however in the graph in Fig. 4(b) follower $i$ is restricted to receiving information from follower $i-1$ only, whereas in Fig. 4(a), it can receive information from both $i-1$ and $i+1$.

We implemented four simulations to compare the results proposed in this paper and also to compare them with the results in [30]. The directed graph in Fig. 4(b) was employed to illustrate Theorem 1 in Simulation 1. In Simulation 2, we implemented the controller designed using Theorem 2 with the undirected graph in Fig. 4(a). We applied Theorem 1 using the same undirected graph in Fig. 4(a) in Simulation 3. In Simulation 4, we applied the event-based control strategy proposed in Theorem
3 [30], also using the directed graph in Fig. 4(b). Out of the results in [30], we chose Theorem 3 for comparison, because it has a way to avoid continuous communications between the followers; this allows for a fair comparison with our methods.

In the first three simulations, we aimed to restrict the predicted upper bound on the tracking error to $\Delta \leq$ 0.05 . In the design, we chose the same $Q$ matrix and adjusted $R$ to obtain the same control gains in the three simulations. The parameters of the triggering conditions (8) and (30) and the design parameters were set as shown in Table 1. In Simulation 4, using the same matrix $Q$, we computed the control gain $K=[2.63,7.24]$ and also chose the parameters required by Theorem 3 of [30] as follows: $h=5, \beta_{1}=0.1, \beta_{2}=0.15, \gamma=0.2$ and $\tau=0$ (see [30] for the definition of these parameters). In all simulations we endeavoured to achieve the least number of communications events.

The simulation results achieved in Simulations 1-3 are shown in Table 1 . In the table, $J_{[18,20]}$ denotes the maximum actual tracking error $\sum_{i=1}^{N}\left\|\varepsilon_{i}(t)\right\|^{2}$ observed over the time interval $t \in[18,20], t_{[0,20]}, t_{[0,10]}, t_{[10,20]}$ and $E_{[0,20]}, E_{[0,10]}, E_{[10,20]}$ represent the minimum interevent intervals and the total number of events occurred in the system on the time intervals $[0,20],[0,10],[10,20]$, respectively. The corresponding results of Simulation 4 are $J_{[18,20]}=1.8778 \times 10^{-6}, t_{[0,20]}=21.1 \mathrm{~ms}, t_{[0,10]}=$ $21.1 \mathrm{~ms}, t_{[10,20]}=101.5 \mathrm{~ms}, E_{[0,20]}=2857, E_{[0,10]}=$ 1767 and $E_{[10,20]}=1090$. The tracking errors are shown in Fig. 5, which illustrates that all four eventtriggered tracking control laws enable all the followers to synchronize to the leader.

The first comparison was made between the techniques developed in this paper for systems connected over directed and undirected graphs; these techniques were applied in Simulations 1 and 2, respectively. Although the minimum inter-event intervals in Simulation 2 were observed to be smaller than those in Simulation 1, on average the events were triggered less frequently in Simulation 2. This demonstrates that connecting the followers into an undirected network and using the design scheme based on Theorem 2 may lead to some advantages in terms of usage of communication resources.

Next, we compared Simulations 2 and 3 using the same undirected follower graph in Fig. 4(a) based on Theorems 1 and 2 developed in the paper. Compared with Simulation 2, more communication events and smaller minimum inter-event intervals were observed in Simulation 3. One possible explanation to this is because the method based on Theorem 2 takes an advantage of the symmetry property of the matrix $\mathcal{L}+G$ of the undirected follower graph in the derivation.

Finally, we compared Simulations 1 and 4 where we 
Table 1

The design parameters and simulation results.

Simulation 1 Simulation 2 Simulation 3

(Theorem $1 \quad$ (Theorem 2 (Theorem 1 and Fig. 4(b)) and Fig. 4(a)) and Fig. 4(a))

\begin{tabular}{cccc}
\hline$Q$ & {$\left[\begin{array}{cc}10.59 & 0.42 \\
0.42 & 1.05\end{array}\right]$} & {$\left[\begin{array}{cc}10.59 & 0.42 \\
0.42 & 1.05\end{array}\right]$} & {$\left[\begin{array}{cc}10.59 & 0.42 \\
0.42 & 1.05\end{array}\right]$} \\
$R$ & 1.1394 & 0.1 & 1.5405 \\
$K$ & {$[5.2313 .08]$} & {$[5.2313 .08]$} & {$[5.2313 .08]$} \\
$\alpha$ & 0.0877 & - & 0.0649 \\
$\omega$ & 0.001 & - & 0.001 \\
$\mu_{i}$ & 0.1 & 0.1 & 0.1 \\
$\sigma_{i}$ & 0.5025 & 0.7198 & 0.3007 \\
$\nu_{i}$ & 2.5 & 2 & 1.2 \\
$\gamma$ & $2.9769 \times 10^{-5}$ & $7.9990 \times 10^{-6}$ & $3.1507 \times 10^{-6}$ \\
$\Delta$ & 0.0462 & 0.0462 & 0.0462 \\
$J_{[18,20]}$ & $3.4524 \times 10^{-7}$ & $3.5259 \times 10^{-6}$ & $1.5518 \times 10^{-6}$ \\
$t_{[0,20]}$ & $6.2 \mathrm{~ms}$ & $5.9 \mathrm{~ms}$ & $1.9 \mathrm{~ms}$ \\
$t_{[0,10]}$ & $6.2 \mathrm{~ms}$ & $5.9 \mathrm{~ms}$ & $1.9 \mathrm{~ms}$ \\
$t_{[10,20]}$ & $30.7 \mathrm{~ms}$ & $24.9 \mathrm{~ms}$ & $26.0 \mathrm{~ms}$ \\
$E_{[0,20]}$ & 2240 & 2091 & 2979 \\
$E_{[0,10]}$ & 1285 & 1217 & 1993 \\
$E_{[10,20]}$ & 955 & 874 & 986 \\
\hline
\end{tabular}

used the same directed follower graph in Fig. 4(b) for both designs. Although compared with the method of Theorem 3 of [30], our method produced smaller minimum time intervals between the events, the total number of events occurred during the simulation using our method was also smaller. We remind that we endeavoured to select the simulation parameters and the controller gains for this simulation to reduce the total number of events. We also tried to compare the performance of the two methods by tuning the controller of [30] to almost the same gain as in Simulation 1 , but the results in Simulation 4 were even worse, producing a much greater number of communication events $\left(E_{[0,20]}=5553, E_{[0,10]}=3100\right.$ and $E_{[10,20]}=$ 2453).

\section{Conclusions}

The paper has studied the event-triggered leaderfollower tracking control problem for multi-agent systems. We have presented sufficient conditions to guarantee that the proposed event-triggered control scheme leads to bounded tracking errors. Furthermore, our results show that by adjusting the parameters of the triggering condition, the upper bound on the tracking errors guaranteed by these conditions can be

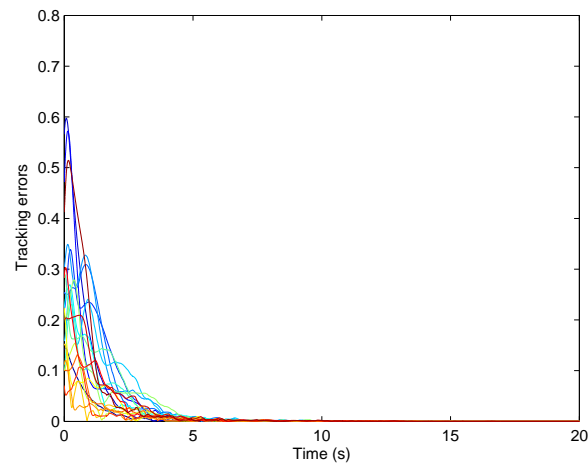

(a) Simulation 1

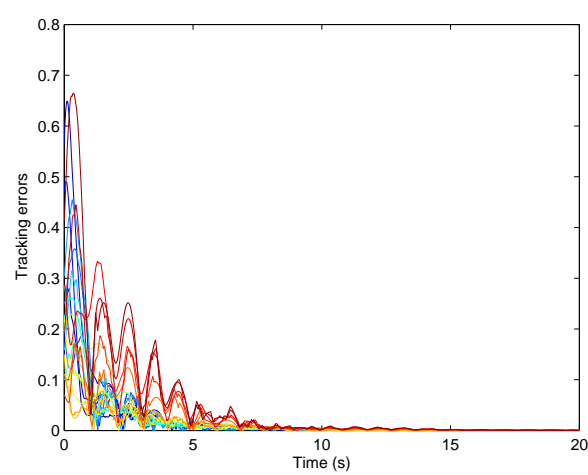

(b) Simulation 2

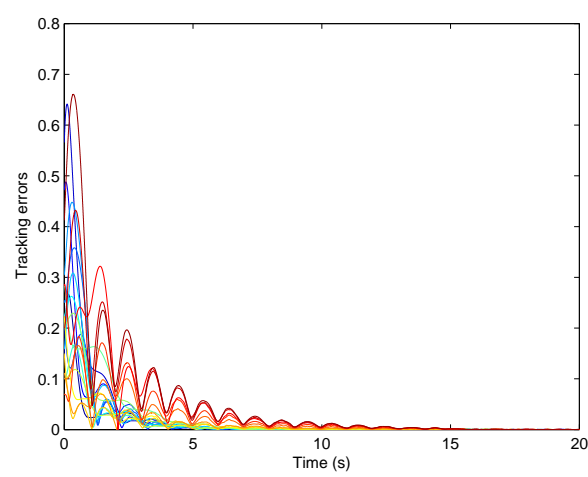

(c) Simulation 3

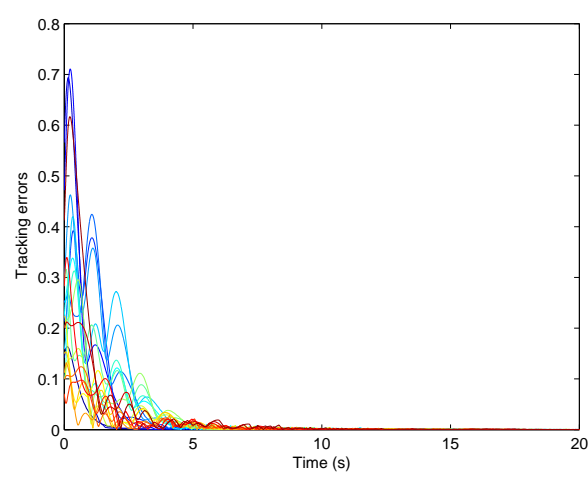

(d) Simulation 4

Fig. 5. Tracking errors $\left\|\varepsilon_{i}\right\|$. 
tuned to a desired small value, at the expense of more frequent communications during an initial stage of the tracking process. Such conditions have been derived for both undirected and directed follower graphs. Also, we showed that the proposed event triggering conditions do not lead to Zeno behavior even if a tight accuracy requirement on the tracking errors is imposed. In fact, with the proposed triggering rules, such tight accuracy requirements do not impact the inter-event intervals after a sufficiently large time. We also presented a computational algorithm which allows the nodes to continuously generate the combinational state at every node which is needed to implement these event triggering schemes. Thus, continuous monitoring the neighboring states is avoided. The efficacy of the proposed algorithm has been demonstrated using a simulation example. Future work will include the study of robustness of the proposed control scheme.

\section{Acknowledgements}

The authors thank the Associate Editor and the Reviewers for their helpful and constructive comments.

\section{References}

[1] W. Ren and R. W. Beard, Distributed consensus in multivehicle cooperative control. London: Springer-Verlag, 2008.

[2] G. Xie, H. Liu, L. Wang, and Y. Jia, "Consensus in networked multi-agent systems via sampled control: fixed topology case," Proc. ACC, 2009, pp.3902-3907.

[3] M. Mazo Jr., A. Anta, and P. Tabuada, "An ISS self-triggered implementation of linear controller," Automatica, 46, 13101314, 2010.

[4] W. P. M. H. Heemels, K. H. Johansson, and P. Tabuada, "An introduction to event-triggered and self-triggered control," Proc. 51st IEEE CDC, 2012, pp.3270-3285.

[5] P. Tabuada, "Event-triggered real-time scheduling of stabilizing control tasks," IEEE Trans. Autom. Contr., 52, 1680-1685, 2007.

[6] J. Lunze and D. Lehmann, "A state-feedback approach to event-based control," Automatica, 46, 211-215, 2010.

[7] D. V. Dimarogonas and K. H. Johansson, "Event-triggered control for multi-agent systems", Proc. 48th IEEE CDC - 28th CCC, 2009, pp.7131-7136.

[8] X. Wang and M. Lemmon, "Event-triggering in distributed networked control systems," IEEE Trans. Autom. Contr., 56, 586-601, 2011.

[9] D. V. Dimarogonas, E. Frazzoli, and K.H. Johansson, "Distributed event-triggered control for multi-agent systems," IEEE Trans. Autom. Contr., 57, 1291-1297, 2012.

[10] W.P.M.H. Heemels and M.C.F. Donkers, "Model-based periodic event-triggered control for linear systems," Automatica, 49, 698-711, 2013.

[11] G. S. Seyboth, D. V. Dimarogonas, and K. H. Johansson, "Event-based broadcasting for multi-agent average consensus," Automatica,49, 245-252, 2013.
[12] Y. Fan, G. Feng, Y. Wang, and C. Song, "Distributed eventtriggered control for multi-agent systems with combinational measurements," Automatica, 49, 671-675, 2013.

[13] X. Meng and T. Chen, "Event based agreement protocols for multi-agent networks," Automatica, 49, 2125-2132, 2013.

[14] W. Zhu, Z. P. Jiang, and G. Feng, "Event-based consensus of multi-agent systems with general linear models," Automatica, 50, 552-558, 2014.

[15] E. Garcia, Y. Cao, and D. W. Casbeer, "Decentralized event-triggered consensus with general linear dynamics," Automatica, 50, 2633-2640, 2014.

[16] D. Liuzza, D. V. Dimarogonas, M. di Bernardo, and K. H. Johansson, "Distributed model-based event-triggered control for synchronization of multi-agent systems," Proc. IFAC Conf. Nonlinear Contr. Syst, 2013, pp. 329-334.

[17] A. Adaldo, F. Alderisio, D. Liuzza, G. Shi, D. V. Dimarogonas, M. di Bernardo and K. H. Johansson, "Eventtriggered pinning control of complex networks with switching topologies," Proc. 53rd IEEE CDC, 2014, pp. 2783-2788.

[18] A. Jadbabaie, J. Lin, and S. A. Morse, "Coordination of groups of mobile autonomous agents using nearest neighbor rules," IEEE Trans. Automat. Contr., 48, 988-1001, 2003.

[19] W. Ren and E. Atkins, "Distributed multi-vehicle coordinated control via local information exchange," Int. J. Robust and Nonlinear Contr., 17, 1002-1033, 2007.

[20] Y. G. Hong, J. P. Hu, and L. X. Gao, "Tracking control for multi-agent consensus with an active leader and variable topology," Automatica, 42, 1177-1182, 2006.

[21] J. Hu, G. Chen, and H. Li, "Distributed event-triggered tracking control of leader-follower multi-agent systems with communication delays," Kybernetika, 47, 630-643, 2011.

[22] Y. Zhang and Y. Hong, "Distributed event-triggered tracking control of multi-agent systems with active leader," Proc. 10th World Congress on Intelligent Control and Automation, Beijing, China, 2012, pp. 1453-1458.

[23] H. Li, X. Liao, T. Huang, and W. Zhu, "Event-triggering sampling based leader-following consensus in second-order multi-agent systems," IEEE Trans. Autom. Contr., 60, 19982003, 2015.

[24] J. Hu, J. Geng and H. Zhu, "An observer-based consensus tracking control and application to event-triggered tracking", Communications in Nonlinear Science and Numerical Simulation, 20, 559-570, 2015.

[25] T. Liu, M. Cao, C. De Persis, and J. M. Hendrickx, "Distributed event-triggered control for synchronization of dynamical networks with estimators", Proc. IFAC Workshop on Distributed Estimation and Control in Networked Systems, Koblenz, Germany, September 2013, pp. 116-121.

[26] M. Franceschelli, A. Gasparri, A. Giua, and C. Seatzu, "Decentralized estimation of Laplacian eigenvalues in multiagent systems," Automatica, 49, 1031-1036, 2013.

[27] H. W. Zhang, Frank L. Lewis, and Z. H. Qu, "Lyapunov, adaptive, and optimal design techniques for cooperative systems on directed communication graphs," IEEE Trans. Industrial Electronics, 59, 3026-3041, 2012.

[28] J. Hu and Y. Hong, "Leader-following coordination of multiagent systems with coupling time delays," Physica A, 374, 853-863, 2007.

[29] E. Kaszkurewicz and A. Bhaya, Matrix Diagonal in Systems and Computation, Birkhäuser Boston, 2000.

[30] W. Zhu and Z.P. Jiang, "Event-based leader-following consensus of multi-agent systems with input time delay," IEEE Trans. Automat. Contr., 60, 1362-1367, 2015. 